\title{
Estudo exploratório da utilização de saw palmetto no tratamento da hiperplasia benigna da próstata por urologistas de Porto Alegre
}

\author{
Gabriela Ferreira, ${ }^{1}$ Mauro Silveira de Castro, ${ }^{1}$ Raquel Bridi ${ }^{*, 2}$
}

${ }^{1}$ Departamento de Produção e Controle de Medicamentos, Faculdade de Farmácia, Universidade Federal do Rio Grande do Sul, Avenida Ipiranga, 2752, 90610-000 Porto Alegre-RS, Brasil,

${ }^{2}$ Departamento de Produção de Matéria-prima, Faculdade de Farmácia, Universidade Federal do Rio Grande do Sul, Avenida Ipiranga, 2752, 90610-000 Porto Alegre-RS, Brasil

\begin{abstract}
RESUMO: O objetivo deste trabalho foi avaliar a utilização de fitoterápicos a base de saw palmetto na terapia sintomática da hiperplasia benigna da próstata (HBP) por médicos urologistas da cidade de Porto Alegre. Consistiu em um estudo transversal, exploratório, por meio de entrevista semi-estruturada aplicada a urologistas de Porto Alegre. A amostra foi obtida utilizando catálogo do plano de saúde UNIMED- Porto Alegre. A randomização foi realizada através de sistemática aleatória, sendo sorteados trinta e cinco médicos, dos quais 21 foram selecionados para realização da pesquisa através de questionário. Todos os urologistas entrevistados avaliam e tratam pacientes com hiperplasia benigna da próstata. $\mathrm{O}$ saw palmetto não foi citado como terapia medicamentosa de $1^{\mathrm{a}}$ ou $2^{\mathrm{a}}$ escolhas no tratamento da HBP. O grupo farmacológico mais freqüentemente utilizado para o tratamento da HBP foi $\propto$-bloqueadores. Mais da metade dos médicos entrevistados relata ter conhecimento sobre a utilização do saw palmetto, principalmente através de artigos científicos. Os resultados indicam que o saw palmetto não é prescrito pelos urologistas em Porto Alegre, todavia a maior parte destes profissionais tem conhecimento sobre sua utilização.
\end{abstract}

Unitermos: Serenoa repens, Arecaceae, saw palmetto, HBP, estudo exploratório, urologistas, Porto Alegre.

\begin{abstract}
Investigation study concerning the saw palmetto use for the benign prostatic hyperplasia treatment by urologists in Porto Alegre". The aim of this work was to assess the prescription of saw palmetto phytomedicines for improving symptoms of benign prostatic hyperplasia $(\mathrm{BPH})$ by urologists in Porto Alegre (Brazil). The study was transversal and exploratory consisting of a semi-structured interview with urologists. The sample was obtained from the UNIMED Catalog - Porto Alegre (Brazil). The randomization was made by chance, being selected thirty-five physicians. The first twenty-one who accepted to participate were interviewed. All the interviewed urologists treat patients with benign prostatic hyperplasia. The saw palmetto was not mentioned as the first or second choice for treating BPH. The $\alpha$-blockers were the most mentioned pharmacological group. However, more than half of urologists have heard about the use of saw palmetto and most of them gathered the information in scientific papers. The results indicate that saw palmetto is not prescribed for urologists in Porto Alegre, although most of them have knowledge about this phytomedicine.
\end{abstract}

Keywords: Serenoa repens, Arecaceae, saw palmetto, BPH, investigated study, urologists, Porto Alegre.

\section{INTRODUÇÃO}

A incidência da hiperplasia benigna da próstata (HBP) aumenta acentuadamente com a idade, atingindo $50 \%$ dos homens com mais de 50 anos e $80 \%$ daqueles com mais de 80 anos. A obstrução do fluxo urinário decorrente da HPB pode acarretar manifestações clínicas relacionadas ao aumento progressivo da próstata. Essa obstrução pode ser causada por dois mecanismos: o estreitamento do lúmen uretral, descrito como obstrução estática e o aumento do tônus da musculatura lisa prostática, chamado de obstrução dinâmica (Burnett \& Wein, 2006; Nickel, 2006; Wilt et al., 2002). Todavia, a etiologia e a patogênese da HBP ainda não estão completamente esclarecidas. Alguns dos principais fatores de risco para o seu desenvolvimento são idade, história familiar e níveis elevados de hormônios sexuais (Arap \& De Goes, 2000; Burnett \& Wein., 2006; Veltri et al., 2002; Zlotta et al., 2005).

O tratamento para HBP está baseado no resultado do International Prostate Symptom Score (IPSS), questionário que mede a severidade dos 
sintomas referentes a obstrução urinária (Suaid et al., 2003). Atualmente existem diversas opções terapêuticas disponíveis, variando desde a terapia medicamentosa, até o tratamento cirúrgico, com a ressecção transuretral da próstata (RTUP) e os procedimentos minimamente invasivos. No Brasil, o custo anual (setembro/2002) do tratamento farmacológico, baseado nos preços de alfa-bloqueadores (tamsulosin, alfuzosin, doxazosin e terazosin) nas mínimas dosagens prescritas foi calculado em US\$ 355 por paciente. O custo da cirurgia de RTUP pela tabela do Sistema Único de Saúde (SUS) foi orçada em US\$ 173 por paciente e pela tabela da Associação Médica Brasileira (AMB) em US\$ 933 dólares incluindo despesas hospitalares (Arap \& De Goes, 2000; Suaid et al., 2003). O principal objetivo do tratamento é obter a melhora dos sintomas. A abordagem terapêutica medicamentosa pode incluir fármacos que reduzem o tônus da musculatura lisa prostática ou as dimensões da próstata. $\mathrm{O}$ uso de fitoterápicos no manejo clínico de pacientes com HBP é largamente utilizado na Europa e ganhou popularidade nos EUA na década de 90, quando houve um grande crescimento na utilização desses produtos. Um dos fitoterápicos mais conhecidos para o tratamento da HBP é o saw palmetto (Serenoa repens L. - Arecaceae) (Arap \& De Goes, 2000; Bent et al., 2006; Martindale, 2004; McPartland \& Pruitt, 2000; Robbers \& Tyler, 1999).

Nos últimos 10 anos registraram-se na base de dados PUBMED (www.pubmed.com) cerca de 30 estudos clínicos comparativos entre produtos a base de saw palmetto, placebo e os principais medicamentos sintéticos utilizados para tratamento da HBP ( $\alpha$-bloqueadores e inibidores da $5 \alpha$-redutase). Parte destes estudos sugere que o saw palmetto apresenta superioridade em relação ao placebo e têm eficácia semelhante aos medicamentos usuais, levando a melhora dos sintomas obstrutivos (intermitência e hesitação ao urinar) bem como irritativos (urgência e noctúria) (Debruyne et al., 2004; Ernest, 2002; Lopatkin et al., 2005; McPartland \& Pruitt, 2000; Willets et al., 2003).

Em estudo realizado em 2002, observou-se que $1,1 \%$ da população adulta dos Estados Unidos, ou aproximadamente 2.5 milhões de adultos, relataram usar saw palmetto na terapia da HBP. Seu uso é aprovado pelo Food and Drug Administration e comumente recomendado como medicamento alternativo nesta doença (Bent et al., 2006; Melo et al., 2002a). Na Europa Ocidental, onde médicos prescrevem produtos fitoterápicos na mesma proporção que medicamentos sintéticos, a terapia baseada em plantas é largamente utilizada por pacientes com sintomas de HBP. Na Itália produtos à base de plantas são prescritos cinco vezes mais freqüentemente que agentes $\propto$-bloqueadores ou finasterida e, na Alemanha, mais de $90 \%$ de todos os medicamentos prescritos para tratamento dos sintomas de HBP envolvem fitoterapia (Marks et al., 2000).

O objetivo deste trabalho foi avaliar a utilização de fitoterápicos a base de saw palmetto na terapia sintomática da HBP por médicos urologistas da cidade de Porto Alegre.

\section{MATERIAL E MÉTODOS}

Foi realizado um estudo transversal, exploratório, por meio de entrevista semi-estruturada aplicada a urologistas de Porto Alegre. Estabeleceu-se como aceitável para o tamanho da amostra a participação de $20 \%$ de urologistas de determinada população, a qual foi definida como a dos médicos urologistas que atendem o plano de saúde UNIMED- Porto Alegre. A população em estudo foi de 107 urologistas. Parar a escolha da amostra, de forma aleatória, aqueles foram enumerados a partir de uma lista ordenada alfabeticamente, sendo sorteados 35, devido a possível recusa de alguns urologistas. Os médicos foram contatados por telefone sempre seguindo a ordem dos números sorteados. A amostra de $20 \%$ da população, composta de 21 indivíduos, foi constituída pelos primeiros médicos que se dispuseram a participar do estudo. Este número só foi possível após serem contatados 29 urologistas. As entrevistas foram realizadas preferencialmente por telefone, sendo que apenas dois médicos solicitaram a entrevista pessoalmente. Este estudo foi aprovado pelo Comitê de Ética em Pesquisa da Universidade Federal do Rio Grande do Sul (n² 2006573).

\section{RESULTADOS E DISCUSSÃO}

A média de faixa etária dos entrevistados foi 45 anos $( \pm 9,0)$, sendo que $91 \%$ pertenciam ao gênero masculino. Quanto à formação acadêmica, 95\% dos entrevistados obtiveram formação acadêmica no estado do Rio Grande do Sul e $28 \%$ possuem curso de mestrado e/ou doutorado. Do total dos entrevistados, $52 \%$ atendem no Sistema Único de Saúde (SUS) e 80\% atendem outros convênios além da Unimed.

Todos os urologistas que contribuíram para a pesquisa avaliam e tratam pacientes com hiperplasia benigna da próstata. Quando questionados sobre a terapia medicamentosa de $1^{\mathrm{a}}$ ou $2^{\mathrm{a}}$ escolhas, o grupo farmacológico mencionado mais freqüentemente foi $\propto$-bloqueador, dentre estes, os fármacos mais citados foram tamsulosina e doxazosina, seguido de inibidores da $5 \propto$-redutase. Nenhum dos urologistas entrevistados citou a utilização de fitoterápicos à base de saw palmetto no tratamento da HBP. Apesar disso, mais da metade relata ter conhecimento sobre a sua utilização. Diversas fontes de informação sobre o saw palmetto foram citadas pelos urologistas, com prioridade para artigos científicos (52\%) e compêndios dos fabricantes (38\%). Outra questão abordada foi a utilização de saw palmetto pelos pacientes sem indicação médica. Verificou-se que a maioria $(81 \%)$ dos médicos entrevistados desconhece se os pacientes fazem uso de fitoterápicos contendo saw 
Tabela 1. Resultado da entrevista aplicada a urologistas de Porto Alegre quanto á utilização do saw palmetto na terapia da HBP (n $=21$ ).

\begin{tabular}{|c|c|c|}
\hline Variável em estudo & & $\operatorname{fr}(\%)^{*}$ \\
\hline \multirow[t]{2}{*}{ Medicamentos mais utilizados na terapêutica da HBP } & $\alpha$-bloqueadores & 100 \\
\hline & Inibidores da $5 \alpha$-redutase & 67 \\
\hline \multirow[t]{2}{*}{ Conhecimento sobre a utilização de saw palmetto na terapia da HBP } & Sim & 95 \\
\hline & Não & 5 \\
\hline \multirow[t]{7}{*}{ Fontes de informação utilizadas } & Artigos científicos & 52 \\
\hline & Compêndios do fabricante & 38 \\
\hline & Livros & 24 \\
\hline & Nenhuma & 5 \\
\hline & Sites na internet & 5 \\
\hline & Médicos estrangeiros & 5 \\
\hline & Relatos de pacientes & 5 \\
\hline \multirow[t]{2}{*}{ Conhecimento da utilização de saw palmetto sem indicação } & $\operatorname{Sim}$ & 19 \\
\hline & Não & 81 \\
\hline
\end{tabular}

* fr $=$ freqüência relativa

palmetto (Tabela 1).

Segundo busca realizada em 10 de julho de 2006 no site da Agência Nacional de Vigilância Sanitária - Anvisa (www.Anvisa.gov.br) encontram-se registrados os seguintes produtos a base de saw palmetto: Prostagem $^{\circledR}$, Permixon ${ }^{\circledR}$, Renopen ${ }^{\circledR}$, Saw Palmetto Brasmed $^{\circledR}, \mathrm{SP}^{\circledR}$, Prosnat ${ }^{\circledR}$, Saw Palmeto ${ }^{\circledR}$, Prostasaw ${ }^{\circledR}$, Prostat $^{\circledR}$, Prostalon $^{\circledR}$, Prostata ${ }^{\circledR}$, Prostalium ${ }^{\circledR}$. De acordo com a RE no 89/ Anvisa de 16 de março de 2004, o saw palmetto faz parte da lista de registro simplificado de fitoterápicos e deve ser vendido sob prescrição médica. Diferentemente dos EUA e da Europa, observamos, por meio deste questionário aplicado especificamente a médicos urologistas, que o saw palmetto não é um dos medicamentos de escolha no tratamento da HBP. E, mesmo não sendo objetivo deste trabalho, verificamos que a maioria dos produtos registrados na Anvisa não se encontra à disposição em farmácias de grandes redes da cidade de Porto Alegre.

Através de revisão sistemática, Wilt et al. (2002), concluíram que o saw palmetto quando comparado a finasterida obteve resposta similar em relação aos sintomas urológicos, as medidas de fluxo urinário e uma menor incidência de efeitos adversos, principalmente relacionados a disfunção erétil. Ainda, Boyle et al. $(2000,2004)$, apesar de algumas limitações encontradas em determinados estudos, verificaram que há fortes evidências de que o Permixon ${ }^{\circledR}$, extrato lipofílico de saw palmetto padronizado em ácidos graxos e esteróis, aumenta o pico de fluxo urinário e reduz os episódios de noctúria quando comparado com placebo. Os efeitos adversos dos extratos de saw palmetto são considerados leves e comparáveis, em termos de incidência, aos efeitos adversos associados ao placebo (Arap \& De Goes, 2000; Schulz et al., 2002). Estudos relatam que a terapia com saw palmetto não tem impacto negativo na função sexual masculina, em contraste com o impacto na função sexual de medicamentos sintéticos como a tamsulosina, a alfusozina e a finasterida, especialmente na ejaculação (Melo et al., 2002b; Zlotta et al., 2005). Em revisão sistemática realizada por Ernst (2002) observa-se que o saw palmetto é superior ao placebo em termos de noctúria e pico de fluxo urinário e semelhante a finasterida em relação a eficácia. Uma metanálise recentemente realizada por Bent et al. (2006) sugere que o saw palmetto induza de fraca a moderada melhora nos sintomas urinários e nas medidas de fluxo. Os autores consideram inúmeras limitações metodológicas, incluindo a curta duração (13 semanas), o insucesso no uso de escores de sintomas validados e indicação do tratamento ocultada em 10 dos 21 estudos avaliados. Ainda, segundo Debruyne et al. (2002), durante a Conferência Internacional de Doenças Urológicas para discutir a HBP e seu tratamento, relatou-se uma falta de estudos clínicos controlados, randomizados, multicêntricos e de longa duração para o saw palmetto.

Considera-se de grande importância o estudo de novos compostos eficazes, de baixo custo e com mínimo de efeitos adversos para o tratamento da HBP. Esta importância esta associada principalmente ao aumento dos custos do tratamento e no crescente envelhecimento da população. Dados apontam que a estimativa de vida da população mundial, incluindo a brasileira, está cada vez maior. Associado a esse fato, existe um aumento no número de pessoas atingidas por doenças relacionadas ao envelhecimento, dentre estas a HBP. Segundo Suaid et al. (2003), o custo dos medicamentos no Brasil é maior a cada ano e a população de risco, homens com idade acima de 40 anos, cresce $25 \%$ a cada ano. Esses fatos predizem que o tratamento da HBP, num futuro próximo, poderá se tornar um problema de saúde pública para a sociedade brasileira, desde que as estimativas de custos anuais permaneçam em torno de 2,3 a 3,4 bilhões de dólares para o grupo submetido a tratamento farmacológico.

Apesar dos inúmeros estudos clínicos existentes utilizando saw palmetto para o tratamento sintomático 
da HBP, do seu amplo uso em países europeus e nos Estados Unidos e do grande número de produtos registrados na Anvisa, os resultados deste estudo indicam que o saw palmetto não é prescrito pelos urologistas de Porto Alegre para o tratamento da HBP. Todavia, grande parte destes profissionais tem conhecimento de sua utilização. A falta de dados sobre as razões que levam os médicos a não prescreverem fitoterápicos a base de saw palmetto, apesar do conhecimento sobre sua utilização, é uma limitação deste estudo. É possível que a existência de resultados controversos, a falta de estudos clínicos de longa duração e o descrédito em produtos de origem vegetal ainda existente em alguns meios da classe médica, desencoraje a utilização destes produtos. Cabe salientar que a utilização de novas terapias medicamentosas, sejam elas de origem vegetal ou sintéticas, somente são justificadas quando existem dados conclusivos sobre eficácia clínica, segurança e qualidade destes produtos. Parecem relevantes novos estudos capazes de identificar as razões que levam os urologistas a não utilizarem saw palmetto e ainda, estudos farmacoepidemiológicos com a finalidade de verificar a indicação desse em balcão de farmácia ou por outros especialistas como os gerontologistas.

\section{REFERÊNCIAS}

Arap S, De Goes PM 2000. Open-ended, non-comparative study to evaluate the efficacy and tolerability of Serenoa repens extract in patients with benign prostatic hyperplasia. J Urol 26: 32-37.

Bent S, Kane C, Shinohara K, Neuhaus J, Hudes ES, Goldberg $\mathrm{H}$, Avins AL 2006. Saw palmetto for benign prostatic hyperplasia. $N$ Engl J Med 354: 557-566.

Boyle P, Robertson C, Lowe F, Roehrborn C 2000. Metaanalysis of clinical trials of permixon in the treatment of symptomatic benign prostatic hyperplasia. Urology 55: 533-539.

Boyle P, Robertson C, Lowe F, Roehrborn C 2004. Updated meta-analysis of clinical trials of Serenoa repens extract in the treatment of symptomatic benign prostatic hyperplasia. BJU Int 93: 751-756.

Burnett AL, Wein AJ 2006. Benign prostatic hyperplasia in primary care: what you need to know. J Urol 175 (3 Pt 2): S19-S24.

Debruyne F, Koch G, Boyle P, Da Silva FC, Gillenwater JG, Hamdy FC, Perrin P, Teillac P, Vela-Navarrete R, Raynaud JP 2002. Comparision of a phytoterapeutic agent (permixon) with an $\alpha$-blocker (tamsulosin) in the treatment of benign prostatic hyperplasia: a 1-year randomized international study. Eur Urol 41: 497-507.

Debruyne F, Boyle P, Da Silva FC, Gillenwater JG, Hamdy FC, Perrin P, Teillac P, Vela-Navarrete R, Raynaud JP, Schulman CC 2004. Evaluation of the clinical benefit of permixon and tamsulosin in severe BPH patients- PERMAL Study Subset Analysis. Prog Urol 14: 326-331.

Ernest E 2002. The risk-benefit profile of commonly used herbal therapies: Gingko, St. John`s Wort, Ginseng,
Echinacea, Saw Palmetto, and Kava. Ann Int Med 136: 42-53.

Lopatkin N, Sivkov A, Walther C, Schlafke S, Medvedev A, Avdeichuk J, Golubev G, Melnik K, Elenberger N, Engelmann U 2005. Long-term efficacy and safety of a combination of sabal and urtica extract for lower urinary tract symptoms - a placebo-controlled, double-blind, multicenter trial. World J Urol 23: 139-146.

Marks LS, Partin AW, Epstein JI, Tyler VE, Simon I, Macairan ML 2000. Effects of a saw palmetto herbal blend in men whith symptomatic benign prostatic hyperplasia. J Urol 163:1451-1456.

Martindale 2004. The Complete Drug Reference. 34.ed. London: Pharmaceutical Press.

McPartland JM, Pruitt PL 2000. Benign prostatic hyperplasia treated with saw palmetto: a literature search and an experimental case study. J Am Osteopath Assoc 100: 87.

Melo EA, Bertero EB, Rios LAS, Junior DM 2002a. Evaluating the efficiency of a combination of Pygeum africanum and stinging nettle (Urtica dioica) extracts in treatment benign prostatic hyperplasia (BPH): double-blind, randomized, placebo controlled trial. Int Braz J Urol 28: 418-425.

Melo EA, Junior DM, Rios LAS 2002b. A double-blind, randomized, placebo-controlled study, to assess the efficacy of alfuzosin in the treatment of patients with benign prostatic hyperplasia. Int Braz J Urol 28: 25-32.

Nickel JC 2006. The overlapping lower urinary tract symptoms of benign prostatic hyperplasia and prostatitis. Curr Opin Urol 16: 5-10.

Robbers JE, Tyler VE 1999. Herbs of Choice: The Therapeutic Use of Phytomedicinals. New York: Haworth Herbal Press.

Schulz V, Hansel R, Tyler VE 2002. Fitoterapia Racional: Um guia de Fitoterapia para as Ciências da Saúde. 4.ed. Barueri: Editora Manole Ltda.

Suaid HJ, Gonçalves MA, Junior AAR, Cunha JP, Cologna AJ, Martins ACP 2003. Estimated costs of treatment of benign prostate hyperplasia in Brazil. Int Braz J Urol 29: 234-237.

Veltri RW, Marks LS, Miller MC, Bales WD, Fan J, Macairan ML, Epstein JI, Partin AW 2002. Saw palmetto alters nuclear measurements reflecting DNA content in men with symptomatic BPH: evidence for a possible molecular mechanism. Urology 60: 617-622.

Willets KE, Clements MS, Champion S, Ehsman S, Eden JA 2003. Serenoa repens extract for benign prostatic hyperplasia: a randomized controlled trial. BJU Int 92: 267-270.

Wilt TJ, Ishani A, Stark G, Mac Donald R, Lau J, Mulrow C 2002. Saw palmetto extracts for treatment of benign prostatic hyperplasia: a systematic review. Cochrane Database Syst Rev (3): CD001423.

Zlotta AR, Teillac P, Raymond JP, Schulman CC 2005. Evaluation of male sexual function in patients with lower urinary tract symptoms (LUTS) associated with benign prostatic hyperplasia (BPH) treated with a phytotherapeutic agent (permixon), tamsulosin or finasteride Eur Urol 48: 269-276. 